\title{
AbORDAGEM PARA MONITORAMENTO TECNOLÓGICO E INVESTIGAÇÃo DE POTENCIAL DE MERCADO: APLICAÇÃO A UMA TECNOLOGIA DE FILAMENTOS NANOESTRUTURADOS PARA IMPRESSORAS DO TIPO FUSED DEPOSITION MODELING
}

Marina Prass Santos (marina.prass@ctnano.org) - Centro de Tecnologia em Nanomateriais e Grafeno, Universidade Federal de Minas Gerais (UFMG).

Glaura Goulart Silva (glaura.silva@ctnano.org) - Centro de Tecnologia em Nanomateriais e Grafeno, Universidade Federal de Minas Gerais (UFMG).

Raoni Barros Bagno (raonibagno@dep.ufmg.br) - Departamento de Engenharia de Produção, Universidade Federal de Minas Gerais (UFMG).

\section{RESUMO}

Estudos de tendências e de prospecção tecnológica tem sido utilizados como ferramenta para informar decisões estratégicas em organizações há décadas. Dentre os métodos consolidados, o Roadmapping se destaca pela sua flexibilidade e apelo gráfico, enquanto a patentometria é uma técnica útil para extrair informações de grandes quantidades de dados. Inspirado no Roadmapping e na patentometria, este estudo propõe uma abordagem de monitoramento tecnológico e investigação de potencial de mercado para um centro tecnológico (CTNano / UFMG), baseado em estudos de patentes e documentos de mercado. As informações obtidas nesses estudos são combinadas em um mapa que apresenta possíveis caminhos de atuação do Centro. O objeto de tal abordagem é uma tecnologia de filamentos poliméricos nanoestruturados para impressoras tipo fused deposition modeling (FDM). No mapa construído foram identificados dois focos potenciais de atuação para o CTNano: (i) tentativa de licenciamento do depósito de patente já efetuado junto a fabricantes de insumos para impressoras FDM que se destacaram nos estudos realizados e (ii) prospecção de projetos envolvendo clientes finais interessados no uso de materiais para manufatura aditiva em contextos de produção industrial, potencialmente aproveitando o posicionamento de mercado conquistado com o licenciamento anterior para ganhar tração nesta iniciativa.

Palavras-chave: análises de futuro para tecnologias; filamentos nanoestruturados; manufatura FDM; patentometria; Roadmapping 


\section{INTRODUÇÃO}

O Centro de Tecnologia em Nanomateriais e Grafeno da Universidade Federal de Minas Gerais (CTNano/ UFMG) é um braço de pesquisa aplicada e extensão tecnológica que reúne pesquisadores com larga experiência em pesquisa e desenvolvimento (P\&D) baseado em materiais dessa classe e que visa servir de ambiente para a contínua transferência de tecnologia a empresas novas ou já estabelecidas. Os esforços do Centro são concentrados na execução de projetos financiados por parceiros, porém eventualmente há margem para desenvolvimento de ideais surgidas dentro da equipe. Foi o caso da tecnologia de filamentos nanoestruturados para impressoras do tipo fused deposition modeling (FDM).

A técnica FDM é uma das muitas que existem no escopo da impressão tridimensional (3D), também chamada manufatura aditiva, na qual objetos são fabricados a partir da adição de material, camada a camada, em contraposição à subtração de material de um bloco base ou à conformação de uma massa inicial. Nas impressoras com tecnologia FDM, especificamente, as peças são fabricadas a partir de polímeros termoplásticos admitidos no formato de filamento (PEREZ; ROBERSON; WICKER, 2014). As aplicações de objetos impressos dependem, em grande medida, das propriedades dos polímeros termoplásticos que constituem os filamentos. Em particular, o uso em peças funcionais e componentes finais requer características de desempenho superiores às dos polímeros tradicionais, por exemplo maior resistência mecânica ou condutividade elétrica. Para alcançá-las, uma rota de pesquisa e desenvolvimento explorada tem sido a aditivação com nanomateriais (WANG et al., 2017). Os nanocompósitos resultantes desse processo, preparados como insumo para impressoras com tecnologia FDM, recebem o nome de filamentos nanoestruturados.

Inicialmente, o desenvolvimento dessa tecnologia no CTNano realizou-se sem financiamento. Após experimentos exploratórios bem-sucedidos, conquistou-se uma parceria com contrapartidas econômicas que resultou em um depósito de patente. Até esse momento, a tecnologia de filamentos nanoestruturados somente gerou custos para o Centro - embora lateralmente, em contexto alternativo, os contatos abertos com a empresa parceira tenham resultado em benefícios - levantando a questão de qual caminho seguir para capturar valor.

Com o intuito de apoiar a tomada de decisão nesse caso, buscaram-se na literatura métodos de prospecção tecnológica e de estudo de tendências para tecnologias portadoras de futuro. No campo da prospecção tecnológica, a patentometria mostra-se bastante útil ao permitir o tratamento de grandes quantidades de dados e o refinamento em tópicos de interesse com o 
emprego de filtros (termos de busca) adequados (FARIA, 2015). Já em relação aos métodos para estudo de tendências de tecnologias portadoras de futuro, o Roadmapping se destaca por permitir conciliar informações de múltiplas fontes em diferentes formatos com forte apelo gráfico, constituindo objetos com elevado poder de síntese e comunicação (PHAAL; FARRUKH; PROBERT, 2004) .

Inspirado no Roadmapping e na patentometria, este estudo propõe uma abordagem de monitoramento tecnológico e investigação de potencial de mercado para o CTNano/ UFMG, com base em estudos de patentes e de documentos de mercado. As informações obtidas nesses estudos são combinadas para composição de um mapa que apresenta ainda possíveis caminhos de atuação identificados para o Centro. A seguir são apresentados pormenores da execução e resultados obtidos em teste piloto com a tecnologia de filamentos nanoestruturados.

\section{METODOLOGIA}

Para construção do mapa que inspirasse possíveis cursos de ação do CTNano, realizaram-se dois estudos: um de patentes e outro de documentos de mercado, em sua maioria relatórios e roadmaps disponíveis publicamente. As informações obtidas a partir desses estudos foram compiladas e conectadas graficamente para composição do mapa, que em seguida levou-se para validação junto a duas lideranças do CTNano. As contribuições dessas lideranças foram usadas para aprimoramento do mapa, conduzindo à versão apresentada na seção de resultados deste artigo. A seguir são detalhadas as etapas de estudo de patentes e de documentos de mercado; depois são apresentados os resultados.

\subsection{Estudo de patentes}

Primeiramente, realizou-se uma coleta de dados no repositório Espacenet. Embora esse serviço online e gratuito esteja associado ao Escritório Europeu de Patentes, ele permite obter informações sobre patentes depositadas e concedidas nos principais escritórios nacionais de propriedade intelectual ao redor do mundo e sobre processos iniciados junto à Organização Mundial de Propriedade Intelectual no âmbito do Patent Cooperation Treaty (PCT).

Como critério inicial de seleção, utilizou-se a seguinte equação de busca: ta = "fused" AND ta = "deposition" AND (ta = "composite" OR ta = "filament" OR ta = "polymer") [idioma: inglês], com o intuito de recuperar desenvolvimentos tecnológicos relacionados a materiais para impressão do tipo FDM. Os dados dos documentos retornados como resultado (patentes e 
modelos de utilidade) foram extraídos em formato de planilha, com colunas para título, nomes dos inventores, nomes dos depositantes, número de publicação, data de prioridade mais antiga, código IPC (international patent classification), código CPC (cooperative patent classification), datas de publicação, data de publicação mais antiga e número da família.

Os documentos foram examinados por leitura do título e, conforme necessário, do resumo, consultado individualmente também na plataforma Espacenet e incorporado à planilha originalmente obtida. A partir desse exame foram selecionados 154 documentos aderentes ao espectro tecnológico de interesse, ou seja, cujo objeto de proteção estivesse relacionado ou ao desenvolvimento de materiais avançados para impressora com tecnologia FDM ou ao desenvolvimento de aplicações com materiais avançados que podem ser usados como insumo em impressoras com tecnologia FDM. Os dados desses documentos foram compilados para informe da maturidade do desenvolvimento tecnológico de interesse a partir número acumulado de depósitos ao longo do tempo (iniciação, crescimento ou estabilização) e identificação (i) de temas recorrentes, (ii) de aspectos relevantes sob a perspectiva do desenvolvimento apoiado em nanomateriais e (iii) de tecnologias concorrentes.

\subsection{Estudo de documentos de mercado}

Nesta etapa foram realizadas buscas com a ferramenta da Google, no site IDTechEx e na base de dados Scopus. Na ferramenta da Google, foram utilizados os seguintes termos de busca: "global 3d printing market report", "mercado de impressão $3 d$ relatório" e "3d printing marketing roadmap", com o intuito de recuperar documentos relacionados à exploração de tendências de futuro para a impressão 3D de modo geral e considerando experiências anteriores na busca de documentos desse tipo. Analogamente, na base de dados Scopus empregou-se a equação de busca "3d print*" AND (foresight OR future OR roadmap). Ao todo analisaram-se 19 documentos, sendo 18 textos e um trecho de vídeo. Um dos textos foi admitido em duas versões complementares e outro fazia referência a dois vídeos em hiperlink, que também foram considerados.

Os documentos foram examinados por leitura completa (e transcrição, no caso do trecho do vídeo) para seleção das informações atinentes e relevantes às tendências projetadas para o mercado de impressão 3D em geral e de manufatura FDM, especificamente. Os destaques de cada documento foram compilados em dossiê e a partir da justaposição de suas informações 
foram definidos nove documentos principais.

\section{RESULTADOS E DISCUSSÃO}

\subsection{Estudo de patentes}

A figura 1 apresenta o número de depósitos de patente relacionados a materiais avançados para manufatura FDM acumulados anualmente de 1999 a 2021, totalizando 154 depósitos. O perfil do gráfico sinaliza que o desenvolvimento de materiais avançados para impressoras com tecnologia FDM está na etapa de crescimento, isto é, já superou a fase de iniciação e ainda não alcançou a de estabilização. Também é interessante notar que o gráfico apresenta uma inflexão por volta de 2013. Quatro anos antes, em 2009, havia expirado a patente de Scott Crump fundador da fabricante de impressoras e filamentos Stratasys - para o método FDM (IMPRESSORA BLOG, 2013). É razoável supor que neste intervalo de tempo a técnica se difundiu consideravelmente, com a oferta de equipamentos e insumos mais baratos, favorecendo o desenvolvimento de materiais avançados e o consequente depósito de patentes em maior número.

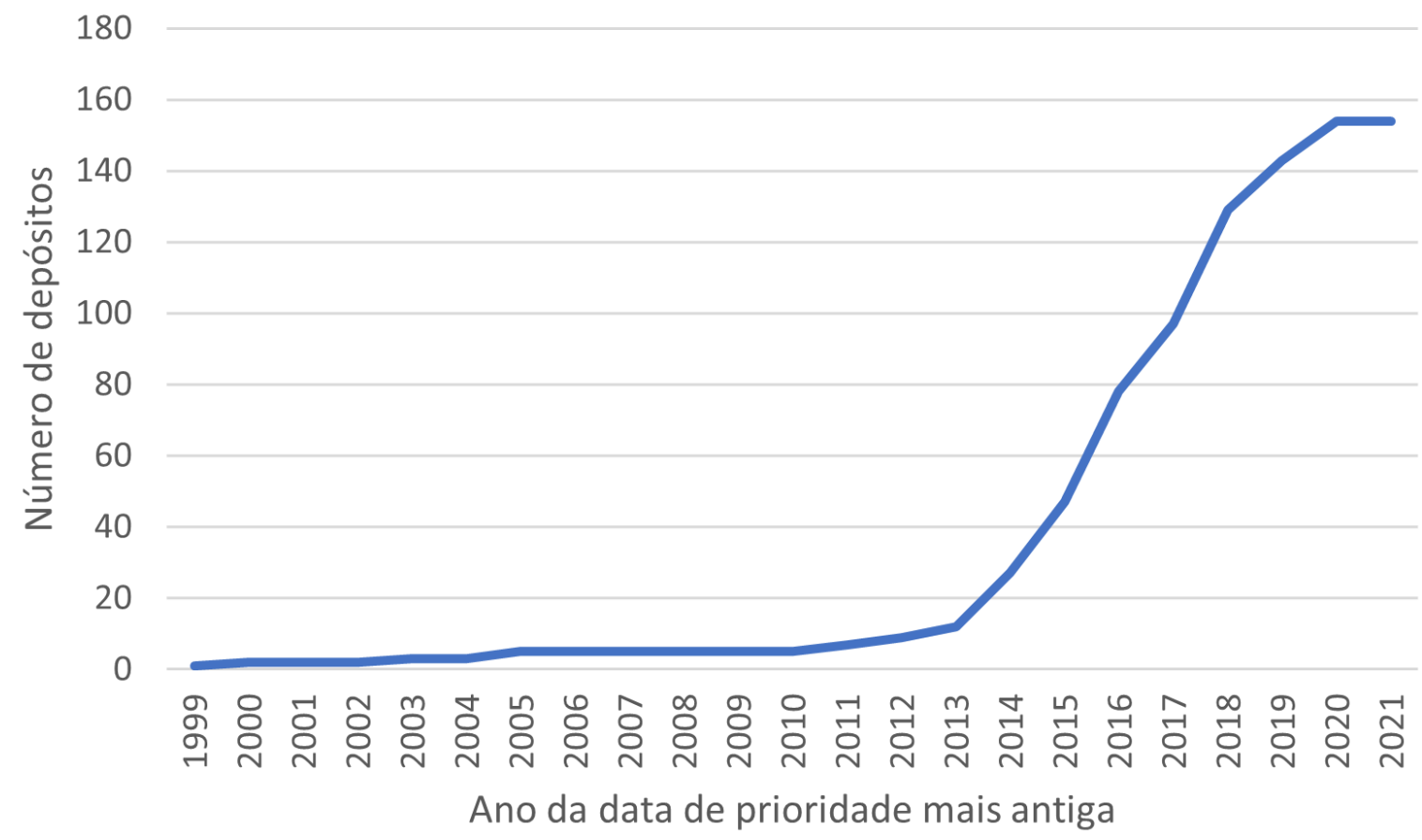

FIGURA 1 - Número de depósitos de patente relacionados a materiais avançados para manufatura FDM acumulados anualmente de 1999 a 2021 . Fonte: os autores 
Em relação aos temas recorrentes, a frequência das menções a materiais com aplicações na área médica - como em endopróteses extensíveis (estentes) e implantes - foi notável, estando presente em 25 dos 154 documentos selecionados. Alguns tipos de aditivos também apareceram de forma marcante; o uso de partículas metálicas ou cerâmicas foi citado em 22 documentos, enquanto nanomateriais foram mencionados em 15 patentes.

Sobre as patentes relacionadas a nanomateriais, elas foram depositadas por 13 organizações diferentes, sendo 11 chinesas, uma japonesa e uma espanhola. Dentre essas organizações estão presentes centros de pesquisa aplicada como o Shanghai National Engineering Research Center for Nanotechnology, universidades como o Beijing Institute of Petrochemical Technology e empresas privadas como a Xerox Corporation. Essas patentes compreendem desenvolvimentos tecnológicos cuja data de prioridade mais antiga varia de 2015 a 2019. Dentre os 15 documentos, quatro patentes envolvem aplicações na área médica (estentes, implantes); quatro citam o uso de PLA como matriz polimérica; três o uso de poliuretano e quatro fazem referência ao uso de grafeno como aditivo. Outros nanoaditivos mencionados são nanotubos de carbono, nano-hidroxiapatita, nanopartículas de ferro e de prata. Algumas patentes não especificam a matriz polimérica e os aditivos empregados no título ou resumo. A justificativa mais frequente para incorporação de nanomateriais é a melhoria de algum aspecto do desempenho mecânico associada a impressão facilitada.

Finalmente, no âmbito de tecnologias concorrentes tiveram destaque materiais avançados para outros tipos consolidados de manufatura aditiva com polímeros, em particular a estereolitografia (SLA - Stereolithography) e a sinterização seletiva a laser (SLS - Selective Laser Sintering), citados em 20 documentos, e desenvolvimentos tecnológicos variados para impressão com matrizes metálicas ou cerâmicas, reportados em 22 documentos. Na identificação de tecnologias concorrentes consideraram-se todos os documentos recuperados pela equação de busca utilizada no Espacenet, e não apenas os documentos selecionados de acordo com os objetos de proteção em foco.

\subsection{Estudo de documentos de mercado}

A tabela 1 indica os nove documentos analisados e definidos como principais, dos quais extraíram-se informações para composição do mapa. 
TABELA 1 - Relação de documentos de mercado analisados e definidos como principais

\begin{tabular}{|c|c|c|}
\hline $\mathrm{N}^{\circ}$ & Documento & $\begin{array}{l}\text { Autores ou organização } \\
\text { responsável }\end{array}$ \\
\hline 1 & $\begin{array}{c}\text { Sample Report 3D Printing Market Analysis and Forecasts from } 2020 \\
\text { to } 2027 \text { (SAMPLE..., 2020d) }\end{array}$ & Grand View Research \\
\hline 2 & $\begin{array}{c}\text { Versão 1: Sample 3D Printing Global Market Analysis, Insights and } \\
\text { Forecast, 2015 - 2026 (SAMPLE..., 2020a) } \\
\text { Versão 2: Sample Report 3D Printing Market Global Analysis, Insights } \\
\text { and Forecast, 2020-2027 (SAMPLE..., 2020b) }\end{array}$ & Fortune Business Insights \\
\hline 3 & Sample Global 3D Printing Market (2020 - 2025) (SAMPLE..., 2020c) & Mordor Intelligence \\
\hline 4 & $\begin{array}{l}\text { Network Based Technology Roadmapping for Future Markets: Case of } \\
\text { 3D Printing (TUCKER et al., 2014) }\end{array}$ & $\begin{array}{l}\text { Katherine Tucker, David } \\
\text { Tucker, James Eastham, } \\
\text { Elizabeth Gibson, Sumir } \\
\text { Varma, Tugrul Daim }\end{array}$ \\
\hline 5 & $\begin{array}{l}\text { Sample Pages 3D Printing and Additive Manufacturing 2020-2030: } \\
\text { COVID Edition (HARROP; COLLINS, 2020) }\end{array}$ & $\begin{array}{l}\text { Jonathan Harrop e Richard } \\
\text { Collins, IDTechEx Research }\end{array}$ \\
\hline 6 & The Future of 3D Printing: Five Predictions (LASELLE, 2020) & Rush LaSelle, Jabil \\
\hline 7 & $\begin{array}{l}100 \text { 3D Printing Experts Predict the Future of 3D Printing in } 2030 \\
\text { (PETCH, 2020) }\end{array}$ & $\begin{array}{l}\text { Michael Petch, 3D Printing } \\
\text { Industry }\end{array}$ \\
\hline 8 & $\begin{array}{l}\text { [Tendências de mercado] Impressora 3D: O que esperar em 2020? } \\
\text { (WISHBOX, 2021) }\end{array}$ & WishBox \\
\hline 9 & $\begin{array}{c}5^{\circ} \text { Webinar PI - A manufatura aditiva (impressão 3D) como aliada da } \\
\text { indústria de plásticos (ARANDA EVENTOS, 2020) }\end{array}$ & Revista Plástico Industrial \\
\hline
\end{tabular}

Fonte: Aranda Eventos (2020), Harrop; Collins (2020), LaSelle (2020), Petch (2020), Sample... (2020a), Sample... (2020b), Sample... (2020c), Sample... (2020d), Tucker et al. (2014), WishBox (2021)

Estima-se que o mercado global de manufatura FDM em 2021 seja da ordem de 832,5 milhões de dólares e que ele deve crescer a uma taxa anual composta (CAGR, do inglês compound annual growth rate) de 15,3\% até 2027 (SAMPLE..., 2020d). A CAGR para o mercado global de impressão 3D em geral, por sua vez, é estimada em 25,7\% até 2026 (SAMPLE..., 2020a), de modo que a tecnologia do tipo FDM deve perder uma fração relativa de mercado (market share). Porém, as projeções indicam que ela ainda será relevante em 2027, com o uso em contextos produtivos tornando-se tão significativo quanto em prototipagem (SAMPLE..., 2020b). Em termos absolutos, o mercado global de impressão 3D deve atingir o valor de 45 bilhões de dólares em 2030 (HARROP; COLLINS, 2020).

Em relação ao cenário base (ano de 2021), destaca-se ainda que avanços na própria tecnologia FDM tem viabilizado o uso de diversos materiais, aumentando assim a adoção generalizada em vários setores (SAMPLE..., 2020c). Investimentos substanciais tem sido realizados por governos pelo mundo nos Estados Unidos, Canadá, Reino Unido, Alemanha, China, Índia e Coreia do Sul. A Coreia do Sul, em particular, estabeleceu um roadmap independente para P\&D em impressão 3D e está fornecendo suporte nacional para executá-lo. O governo está introduzindo incentivos fiscais e acelerando acordos regulatórios da indústria para encorajar a adoção da impressão 3D (SAMPLE..., 2020a). Essas informações são coerentes com o 
desenvolvimento tecnológico de materiais avançados para manufatura FDM em crescimento observado no estudo de patentes.

Outro fator importante no cenário base é o surgimento de insumos para manufatura SLA com resistência mecânica aprimorada (ARANDA EVENTOS, 2020), sinalizando um aumento da concorrência para filamentos poliméricos nanoestruturados, que também se propõem a aumentar o desempenho mecânico das peças impressas. Por outro lado, identificaram-se possíveis aliados na exploração comercial da tecnologia de filamentos nanoestruturados: Ultimaker Material Alliance e Braskem.

A Ultimaker Material Alliance é uma organização que reúne cerca de 80 fornecedores de filamentos e oferece mais de 100 materiais ao mercado, com nível industrial para aplicações industriais e foco em atender às aplicações desejadas pelos clientes finais (desenvolvimento dirigido). Dentre os participantes encontram-se grande empresas como Basf e Ensinger, desenvolvedora de uma poliamida reforçada com fibra de vidro (WISHBOX, 2021).

A Braskem, por sua vez, está ativa no desenvolvimento de materiais avançados para FDM, como comentou Fabio Lamon, gerente global de inovação e tecnologia para manufatura aditiva na empresa, em webnário da Revista Plástico Industrial:

Quando investigamos os 20, 30 anos de impressão 3D identificamos materiais de engenharia para algumas aplicações e consolidação de alguns materiais. Mas a gente também aprendeu que adoção e aceleração passava por desempenho, competitividade e o uso em aplicações high-end, aplicações onde se busca um desempenho melhor. Então a gente começou nossa jornada pelo polipropileno (PP), pelo balanço de propriedades que ele tem, e ele começa a responder a algumas necessidades, e a partir dele a gente já tem esforços em desenvolvimento que visam modificar esse produto para que se possa levar para a cadeia produtos com a utilização de cargas, fibras. A gente fala muito, já ouvi falar de materiais com fibra de carbono, com fibra de vidro, então nós também estamos atentos a essa tendência, já fazendo trabalhos que visam explorar o uso desses sistemas, combinando PP com outros polímeros, PP com outros materiais a fim de viabilizar soluções de alto desempenho e poder continuar contribuindo com a adoção (ARANDA EVENTOS, 2020, 1:02:38 a 1:04:22).

Em direção ao futuro, diferentes fontes sustentam a ideia de que materiais para manufatura aditiva são tanto um forte direcionador de mercado quanto um atributo de produto principal, pois são novos materiais que habilitam novas aplicações e, portanto, abrem caminho para adoção desse grupo de tecnologias em novos contextos (LASELLE, 2020; PETCH, 2020).

Finalmente, em relação ao horizonte de tempo mais longínquo considerado neste estudo, o ano de 2030, algumas tendências devem ser pontuadas. A extrusão com termoplásticos deve continuar dominante entre consumidores finais, isto é, entre usuários que demandam pequena 
escala, como os domésticos e pequenos empreendedores que produzem artigos de decoração e colecionáveis (HARROP; COLLINS, 2020). Porém, em relação ao uso da manufatura aditiva como tecnologia de fabricação em contextos industriais, muitos especialistas acreditam que outras tecnologias de impressão 3D de alto desempenho devem sobressair, em particular aquelas baseadas em matrizes metálicas (PETCH, 2020). Estima-se que em 2030 10\% ou mais de todas as partes produzidas no mundo serão impressas e que a manufatura aditiva será uma grande aliada no estabelecimento de modos de produção e distribuição mais sustentáveis, isto é, que consumam menos energia, emitam menos poluentes e gerem menos lixo. A produção será cada vez mais customizada e próxima do local de consumo, diminuindo a necessidade de estoques ao mesmo tempo em que os tempos de entrega são reduzidos (PETCH, 2020). Também se projeta que as fábricas tornar-se-ão cada vez mais inteligentes e automatizadas, com a manufatura aditiva sendo integrada aos processos produtivos com o apoio de tecnologias como internet das coisas e inteligência artificial.

\subsection{Mapa}

A figura 2 apresenta o mapa construído. Além das camadas para informações dos estudos de patentes e de documentos de mercado, introduziu-se uma terceira para apresentar possíveis caminhos de atuação do CTNano; seu posicionamento intermediário está relacionado ao papel desempenhado pelo Centro como ambiente de transferência tecnológica. Os marcos temporais foram definidos a partir do cenário base e dos elementos de maior destaque no estudo de documentos de mercado. Essa versão já incorpora comentários tecidos pelas lideranças do CTNano na etapa de validação.

Considerando os fatores favoráveis e desfavoráveis levantados no presente e projetados para o futuro, o CTNano pode focar seus esforços em dois núcleos sequenciais:

(i) em relação à tecnologia de filamentos nanoestruturados que já possui um depósito de patente: esse recurso pode ser usado como elemento de portfólio para estabelecer diálogo com grandes fabricantes de polímeros com grades (variedades) para impressão FDM, como a Braskem, ou, mais especificamente, com fabricantes de filamentos relevantes no cenário internacional, como a Ultimaker. Neste último caso, a Ultimaker Material Alliance pode ser um canal efetivo para licenciamento da tecnologia e inserção definitiva do CTNano no mercado da manufatura aditiva, viabilizando a captura de valor dos esforços em $\mathrm{P} \& \mathrm{D}$ já realizados no Centro em um cenário de tempo mais curto, por 
exemplo um a dois anos, e o aproveitamento de tendências projetadas até 2027. É nesta atividade que o Centro deveria focar de maneira mais imediata.

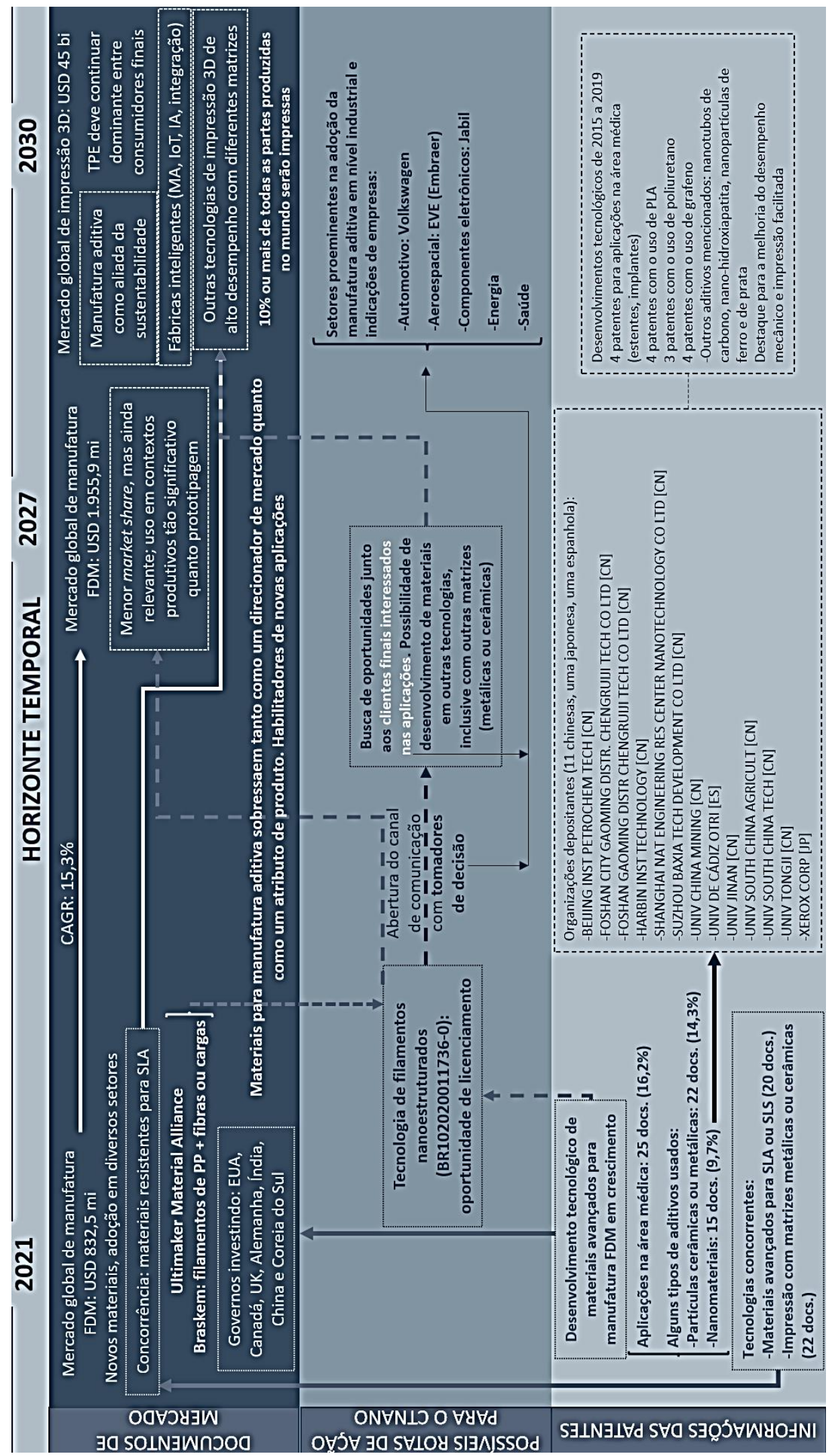

FIGURA 2 - Mapa construído a partir dos estudos de patentes e de documentos de mercado. Fonte: os autores 
(ii) em relação à manufatura aditiva de modo mais geral: é importante considerar que a tecnologia FDM pode não ser a vencedora no contexto de produção industrial e das aplicações que requerem elevado desempenho funcional. O desenvolvimento de tecnologias substitutas pode comprometer a relevância do FDM no médio/longo prazos ou aprisioná-lo em algumas aplicações particulares nas quais ele permaneça como solução mais competitiva. Esse é um aspecto que pode ser analisado com maior profundidade, em complemento aos estudos já realizados. Porém, mesmo com as informações já levantadas entende-se que o CTNano deve, tanto quanto possível, buscar oportunidades de desenvolvimento de materiais a partir da interação com clientes finais, isto é, com as empresas que de fato podem vir a usar os materiais de interesse no contexto industrial, e não apenas os fabricantes dos insumos, como indicado no primeiro núcleo de ação. Desse modo, o Centro estará exposto à possibilidade de capturar valor contido em outras tecnologias de manufatura aditiva. Espera-se que a inserção no mercado proposta no item (i) facilite esse envolvimento com clientes finais, na medida em que abra canais de acesso aos tomadores de decisão nas empresas usuárias. Sob essa perspectiva, o CTNano atuaria como originador do desenvolvimento tecnológico que poderia ser compartilhado com ou licenciado para fabricantes de insumo e comercializado para o cliente final - e, a depender do papel estratégico, para outras empresas do mesmo setor. Nesse caso, os esforços necessários poderiam ser iniciados em um horizonte de curto ou médio prazo com a intenção de se desenrolar no longo prazo, de modo a se aproveitar das tendências projetadas para 2030.

Ilustrando quem podem ser esses clientes finais ou empresas usuárias, os setores proeminentes para adoção da impressão 3D em nível industrial citados com maior frequência nos documentos analisados são: automotivo, aeroespacial, de componentes eletrônicos, de energia e de cuidados com a saúde. No setor automotivo, a Volkswagen está trabalhando junto à HP com manufatura aditiva em escala, e pretende chegar a imprimir 100.000 peças por ano (SAMPLE..., 2020c). No setor aeroespacial, a Eve é uma subsidiária da Embraer que já recebeu 250 encomendas de um veículo elétrico de decolagem e pouso vertical, o eVTOL (Vertical Take-Off and Landing) (SANT'ANNA, 2021) e potencialmente se beneficiará de componentes impressos de alto desempenho e pouco peso. No setor de componentes eletrônicos, a Jabil tem a manufatura aditiva como dimensão estratégica do negócio (LASELLE, 2020). 


\section{CONCLUSÃO}

Neste trabalho uma abordagem de monitoramento tecnológico e investigação de potencial de mercado customizada para o CTNano/ UFMG foi proposta e confrontada pela primeira vez com a tecnologia de filamentos poliméricos nanoestruturados. Como foco de ação mais imediata do Centro em relação à tecnologia, defendeu-se a tentativa de licenciamento junto a fabricantes de insumos para impressoras com tecnologia FDM que se destacaram no estudo de documentos de mercado.

Esse teste preliminar da abordagem sugere que para embasar a tomada de decisão sobre uma tecnologia bastante específica é necessário explorar aspectos mais amplos do espectro tecnológico e do mercado em questão, e quanto mais longo o horizonte de tempo, maior a amplitude dos aspectos a serem considerados. Nesse sentido, uma oportunidade de trabalho futuro é a categorização dos aspectos de cada camada do mapa (patentes, caminhos de atuação e mercado) visando sistematizar a abordagem e assim torná-la mais útil como ferramenta para tomada de decisão bem-informada.

\section{AGRADECIMENTOS}

Agradecemos a Vinícius Gomide e Raissa Guerra pelas gentis contribuições em relação ao mapa elaborado.

\section{REFERÊNCIAS}

ARANDA EVENTOS. $\mathbf{5}^{\circ}$ Webinar PI - a manufatura aditiva (impressão 3D) como aliada da indústria de plásticos. São Paulo, 2020. 1 vídeo (1:02:12 a 1:09:52). Disponível em: https://youtu.be/9NVHgIR2A5s. Acesso em: 20 jun. 2021.

FARIA, L. Análise do desenvolvimento tecnológico a partir de documentos de patentes: um estudo de caso em processos de biolixiviação. 27 nov. 2015. Dissertação (Mestrado em Engenharia de Materiais) - Rede Temática em Engenharia de Materiais, Universidade Federal de Ouro Preto. Ouro Preto, 2015.

HARROP, J.; COLLINS, R. Sample pages 3D printing and additive manufacturing 2020-2030: COVID edition. 2020. Disponível em: https://www.idtechex.com/en/research-report/3d-printing-andadditive-manufacturing-2020-2030-covid-edition/765. Acesso em: 20 jun. 2021.

IMPRESSORA.BLOG. Fim das patentes pode impulsionar o mercado de impressão 3D. 2013. Disponível em: http://www.impressora.blog.br/fim-das-patentes-impulsiona-mercado-de-impressao-3d/. Acesso em: 20 jun. 2021.

LASELLE, R. The Future of 3D printing: five predictions. 2020. Disponível em: https://www.jabil.com/blog/future-of-3d-printing-additive-manufacturing-looks-bright.html. Acesso em: 20 jun. 2021.

PHAAL, R.; FARRUKH, C. J. P.; PROBERT, D. R. Technology roadmapping - a planning framework for evolution and revolution. Technological Forecasting and Social Change, v. 71, p. 5-26, 2004.

PEREZ, A.; ROBERSON, D.; WICKER, R. Fracture surface analysis of 3D-printed tensile specimens of novel ABS-based materials. Journal of Failure Analysis and Prevention, v. 14, n. 3, p. 343-353, 2014. 
PETCH, M. 100 3D Printing experts predict the future of 3D printing in 2030. 2020. Disponível em: https://3dprintingindustry.com/news/100-3d-printing-experts-predict-the-future-of-3dprinting-in-2030-167623/. Acesso em: 20 jun. 2021.

SAMPLE 3D printing global market analysis, insights and forecast, 2015 - 2026. 2020a. Disponível em: https://www.fortunebusinessinsights.com/industry-reports/3d-printing-market-101902. Acesso em: 20 jun. 2021.

SAMPLE report 3D printing market global analysis, insights and forecast, 2020-2027. 2020b. Disponível em: https://www.fortunebusinessinsights.com/industry-reports/3d-printing-market-101902. Acesso em: 20 jun. 2021.

SAMPLE global 3D printing market (2020 - 2025). 2020c. Disponível em: https://www.mordorintelligence.com/industry-reports/3d-printing-market. Acesso em: 20 jun. 2021.

SAMPLE report 3D printing market analysis and forecasts from 2020 to 2027. 2020d. Disponível em: https://www.grandviewresearch.com/industry-analysis/3d-printing-industry-

analysis\#: :text=Report\%20Overview, 8.04\%20million\%20units\%20by\%202027. Acesso em: 20 jun. 2021.

SANT'ANNA, I. Como o "carro voador" da Eve transforma a ficção em realidade e recoloca a Embraer na vanguarda do setor aéreo. 2021. Disponível em: https://www.seudinheiro.com/2021/colunistas/seu-mentor-deinvestimentos/carro-voador-eve-embraer-ivan-santanna/?xpromo=XV-MI-SD-NL-BSD-X-X-MANHA-X-

$\underline{X \& u t m \_s o u r c e=S D \& u t m \_ \text {medium }=X \& u t m \_ \text {campaign }=X V-M I-S D-N L-B S D-X-X-}$

MANHA-X-X\&e=bWFyaW5hcHJhc3NAZ21haWwuY29t. Acesso em: 20 jun. 2021.

TUCKER, K.; TUCKER, D.; EASTHAM, J.; GIBSON, E.; VARMA, S.; DAIM, T. Network based technology roadmapping for future markets: case of 3D printing. Technology and Investment, v. 05, n. 03, p. 137-156, 2014.

WANG, X.; JIANG, M.; ZHOU, Z.; GOU, J.; HUI, D. 3D printing of polymer matrix composites: a review and prospective. Composites Part B: Engineering, v. 110, p. 442-458, 2017.

WISHBOX TECHNOLOGIES. [Tendências de mercado] impressora 3D: o que esperar em 2020? 2021. Disponível em: https://www.wishbox.net.br/blog/tendencias-de-mercado-impressora-3d/\#btn-continuar-lendo. Acesso em: 20 jun. 2021. 